\title{
Spin-locking and cross-polarization under magic-angle spinning of uniformly labeled solids
}

\author{
Ivan Hung and Zhehong Gan * \\ Center of Interdisciplinary Magnetic Resonance, National High Magnetic Field Laboratory, 1800 East Paul Dirac \\ Drive, Tallahassee, FL 32310, USA \\ * Corresponding author. Fax +1 8506441366. \\ E-mail address: gan@magnet.fsu.edu (Z.Gan).
}

\begin{abstract}
Spin-locking and cross-polarization under magic-angle spinning are investigated for uniformly ${ }^{13} \mathrm{C}$ and ${ }^{15} \mathrm{~N}$ labeled solids. In particular, the interferences from chemical shift anisotropy, and ${ }^{1} \mathrm{H}$ heteronuclear and ${ }^{13} \mathrm{C}$ homonuclear dipolar couplings are identified. The physical origin of these interferences provides guidelines for selecting the best ${ }^{13} \mathrm{C}$ and ${ }^{15} \mathrm{~N}$ polarization transfer $r f$ fields. Optimal settings for both the zero- and double-quantum cross-polarization transfer mechanisms are recommended.
\end{abstract}

Keywords: spin-lock, cross-polarization, magic-angle spinning, rotary resonance, recoupling, solid-state, SPECIFIC CP, Hartmann-Hahn, Lee-Goldburg, proteins, decoupling, coherence transfer

\section{Introduction}

Spin-locking is a phenomenon known since the early days of NMR. By applying a continuous-wave $(\mathrm{CW})$ radio-frequency $(r f)$ field, spin polarization projected on to the $r f$ field can be 'locked' in the presence of inhomogeneous fields and other spin interactions [1]. The locked magnetization can be maintained for a relatively long time and decays with a time constant $T_{1 \rho}$, the spin-lattice relaxation time in the rotating frame. Spin-locking is useful to many NMR experiments, in particular, the simultaneous application of spin-lock pulses to two different spin species, $I$ and $S$, enables mutual polarization transfer when their spin-lock $r f$ field amplitudes $\left(v_{1}\right)$ match the so-called Hartmann-Hahn $(\mathrm{HH})$ condition [2], $v_{1 I}=v_{1 S}$, a process commonly known as cross-polarization (CP) [3]. The transfer of polarization from abundant 
nuclei with high gyromagnetic ratios $(\gamma)$ like ${ }^{1} \mathrm{H}$ to nuclei with lower $\gamma$ nuclei $\left(\right.$ e.g.,${ }^{13} \mathrm{C}$ and ${ }^{15} \mathrm{~N}$ ) via $\mathrm{CP}$ is widely used for sensitivity enhancement [4]. Furthermore, CP with short contact times can be used for selective coherence transfer, for instance, it is the most commonly used method for establishing heteronuclear correlation (HETCOR) between ${ }^{13} \mathrm{C}$ and ${ }^{1} \mathrm{H}$ in solids. In the case of uniformly ${ }^{13} \mathrm{C}$ and ${ }^{15} \mathrm{~N}$ labeled proteins, $\mathrm{CP}$ between ${ }^{13} \mathrm{C}$ and ${ }^{15} \mathrm{~N}$ with low $r f$ fields can be used for coherence transfer between specific spin pairs to take advantage of the large ${ }^{13} \mathrm{C}$ and ${ }^{15} \mathrm{~N}$ chemical shift ranges, namely SPECIFIC CP [5, 6]. The SPECIFIC CP technique is a basic building block used in many multi-dimensional experiments developed for spectral assignment and structural elucidation of uniformly labeled proteins. The efficiency of ${ }^{13} \mathrm{C} \leftrightarrow{ }^{15} \mathrm{~N}$ SPECIFIC $\mathrm{CP}$ transfer is mostly determined by two factors, the $T_{1 \rho}$ of the two spin species and accurate matching of the $\mathrm{CP}$ condition within the magnitude of the ${ }^{13} \mathrm{C}-{ }^{15} \mathrm{~N}$ dipolar coupling. The importance of matching $\mathrm{CP}$ conditions has been examined extensively, but the spin-lock near the conditions commonly used for ${ }^{13} \mathrm{C} \leftrightarrow{ }^{15} \mathrm{~N}$ SPECIFIC CP has to our knowledge not been investigated in detail; specifically, the interference effects from chemical shift anisotropy (CSA), and hetero- and homo-nuclear dipolar interactions. This study was motivated by the fact that both the spin-lock and cross-polarization efficiencies directly impact the sensitivity of many solid-state NMR experiments applied to proteins and biomolecules.

High-resolution solid-state NMR requires magic-angle spinning (MAS) of the sample to average line broadening from anisotropic spin interactions. Modulation by MAS can affect spinlocking and cross-polarization performance. In particular, it is well-known that the $\mathrm{HH}$ condition under MAS is modified into sidebands $v_{1 I}-v_{1 S}=n \cdot v_{\mathrm{r}}$, where $v_{\mathrm{r}}$ is the MAS frequency [7-10]. Further, fast MAS can cause partial breakdown of the proton 'spin bath,' making the four primary HH matching conditions $n= \pm 1, \pm 2$ narrower than the single match condition observed 
for static samples. CP is usually mediated by spin flip-flop $\left(I_{+} S_{-}+I S_{+}\right)$, or the so-called zeroquantum (ZQ) dipolar Hamiltonian, but under MAS, flip-flip/flop-flop $\left(I S S_{-}+I_{+} S_{+}\right)$can also occur via the double-quantum (DQ) dipolar Hamiltonian mechanism at the conditions $v_{1 I}+v_{1 S}=$ $n \cdot v_{\mathrm{r}}$, which can often be met under fast MAS and weak spin-locking $r f$ fields [10-13]. In order to compensate mismatch of the $\mathrm{CP}$ conditions due to inhomogeneous $r f$ fields, a sweep of the $r f$ field amplitude [14-16] or frequency offset [17-19] across one of the matching conditions is usually applied to improve CP efficiency [14-16]. More sophisticated pulse schemes have been developed as well by taking the MAS modulation into consideration [20, 21].

This study focuses mainly on the spin-lock of ${ }^{13} \mathrm{C}$ and ${ }^{15} \mathrm{~N}$ nuclei under MAS. Modulation of the various spin interactions under MAS can have a large effect on spin-locking depending on the applied $r f$ field strength. Since relatively long contact times are typically needed for CP between weakly-coupled ${ }^{13} \mathrm{C}$ and ${ }^{15} \mathrm{~N}$ spin pairs, even small interferences from high-order effects can cause a dramatic loss of spin-lock and coherence transfer efficiency. Under MAS, the spatial part of second-rank anisotropic spin interactions consists of $v_{\mathrm{r}}$ and $2 v_{\mathrm{r}}$ modulation components. A reintroduction of the interactions under MAS, often called recoupling (or rotary resonance) [2224], can occur when the nutation frequency of the spin part due to the $r f$ field matches the modulation frequency of the spatial components, i.e., once or twice the spinning frequency. Both ${ }^{13} \mathrm{C}$ and ${ }^{15} \mathrm{~N}$ can have large CSA and the rotary resonance can cause rapid decay of the polarization during spin-locking. Hence, $r f$ fields near the primary resonance conditions $v_{1 S}=n \cdot v_{\mathrm{r}}$ $(n=1,2)$ should always be avoided.

For ${ }^{13} \mathrm{C}$ and ${ }^{15} \mathrm{~N}$ experiments, the strong heteronuclear dipolar coupling to protons needs to be decoupled during the spin-lock. The ${ }^{13} \mathrm{C} /{ }^{15} \mathrm{~N}$ spin-lock $r f$ fields should be set far from the ${ }^{1} \mathrm{H}-S \mathrm{CP}$ conditions $\left(S={ }^{13} \mathrm{C}\right.$ or $\left.{ }^{15} \mathrm{~N}\right)$ under MAS, $v_{1 S}=v_{1 \mathrm{H}}-m \cdot v_{\mathrm{r}}$. It will be shown here that high- 
order $\mathrm{CP}$ conditions with $m$ up to 5 from the proton decoupling can cause signal loss during the long spin-lock used in $\mathrm{CP}$. The ${ }^{13} \mathrm{C} /{ }^{15} \mathrm{~N} r f$ fields at these conditions extend down to the range commonly used for ${ }^{13} \mathrm{C} \leftrightarrow{ }^{15} \mathrm{~N}$ SPECIFIC CP, therefore, this effect needs to be considered carefully.

For uniformly labeled proteins, homonuclear dipolar couplings among the ${ }^{13} \mathrm{C}$ spins are present. The nutation of the homonuclear dipolar Hamiltonian under the spin-lock $r f$ field is more complicated due to the bilinear nature of the spin operators and the large ${ }^{13} \mathrm{C}$ chemical shift ranges of coupled spins. In the case without any frequency offset, the nutation frequency of the the homonuclear dipolar Hamiltonian is equal to twice the $r f$ field. Thus, recoupling of the homonuclear dipolar interaction occurs at $v_{1 S}=n \cdot v_{\mathrm{r}}(n=1 / 2,1)$, in contrast to the $n=1,2$ conditions for CSA and heteronuclear dipolar coupling. The $n=1 / 2$ condition is unique to homonuclear dipolar coupling and commonly known as the HORROR condition [25, 26]. The

${ }^{13} \mathrm{C}$ homonuclear recoupling is complicated further by frequency offsets from chemical shift. As it will be illustrated with samples of uniformly ${ }^{13} \mathrm{C}$ and ${ }^{15} \mathrm{~N}$ labeled alanine and GB1 protein, interferences and signal loss can occur at various ${ }^{13} \mathrm{C} r f$ field amplitudes depending on the chemical shift offsets to the spin-lock $r f$ frequency. The occurrences of all resonance conditions mentioned above including those due CSA, heteronuclear dipolar coupling to ${ }^{1} \mathrm{H}$ and homonuclear coupling amongst the ${ }^{13} \mathrm{C}$ nuclei need to be considered when selecting the optimal $r f$ field for ${ }^{13} \mathrm{C} \leftrightarrow{ }^{15} \mathrm{~N}$ coherence transfer via either the ZQ or DQ CP mechanism.

\section{Experimental}

The samples of $\left[{ }^{15} \mathrm{~N}\right]$ alanine and $\left[{ }^{13} \mathrm{C},{ }^{15} \mathrm{~N}\right]$ alanine were purchased from Cambridge Isotope Laboratories, Inc. (CIL) and used without further purification. The sample of $\left[{ }^{13} \mathrm{C}\right.$, 
${ }^{15}$ N]GB1 protein was kindly provided by Prof. Conggang Li from the Wuhan Institute of Mathematics and Physics, China. All experiments were performed on a $18.8 \mathrm{~T} 63 \mathrm{~mm}$ mediumbore superconducting magnet with a Bruker Avance III HD $800 \mathrm{MHz}$ spectrometer and a Low-E $3.2 \mathrm{~mm}$ MAS probe [27] designed and built at the NHMFL. Samples were packed into 'thinwall' Pencil style rotors using the complete nominal volume of $36 \mu \mathrm{L}$, i.e., no extra spacers were used to restrict the sample volume. The ${ }^{13} \mathrm{C}$ and ${ }^{15} \mathrm{~N}$ spin-locking experiments were acquired using the pulse sequence shown in Fig. 1 by varying the $r f$ field $v_{1 S}$ during the spin-lock pulse in steps of $v_{\mathrm{r}} / 100$. For $\left[{ }^{15} \mathrm{~N}\right]$ alanine, $\left[{ }^{13} \mathrm{C},{ }^{15} \mathrm{~N}\right]$ alanine and $\left[\mathrm{U}-{ }^{13} \mathrm{C},{ }^{15} \mathrm{~N}\right] \mathrm{GB} 1,8,4$ and 4 transients were averaged for each $v_{1 S}$ step, respectively. For all measurements, the spin-lock pulse was fixed to 67 rotor periods $(\sim 5 \mathrm{~ms})$ at a MAS frequency of $v_{\mathrm{r}}=13.5 \mathrm{kHz} .{ }^{1} \mathrm{H}$ SPINAL-64 decoupling [28] with $83 \mathrm{kHz}$ rf field was used during acquisition.

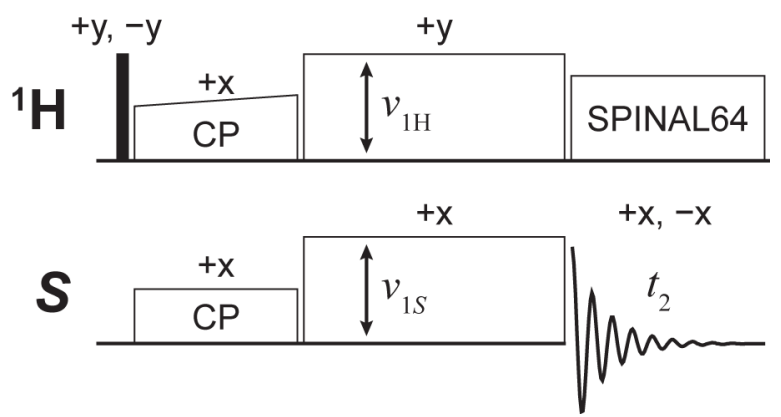

Fig. 1 Pulse sequence used to map the spin-lock efficiency under MAS, where $v_{1 S}$ and $v_{1 \mathrm{H}}$ are the applied $r f$ fields on the $S$ and ${ }^{1} \mathrm{H}$ nuclei during the spin-lock period, respectively.

\section{Results and Discussion}

The signal intensities by varying the $r f$ field strength $v_{1 S}$ of a $\sim 5 \mathrm{~ms}$ spin-lock were measured for alanine. The results are plotted in Fig. 2 in units of the spinning frequency $v_{\mathrm{r}}$. The $\mathrm{NH}_{3}$ curves (Fig. 2a and 2e) for the $\left[{ }^{15} \mathrm{~N}\right]$ and $\left[{ }^{15} \mathrm{~N},{ }^{13} \mathrm{C}\right]$ labeled samples are essentially the same with dips at $v_{1 S}=n \cdot v_{\mathrm{r}}(n=1,2)$, the main rotary resonance conditions for CSA. $\mathrm{NH}_{3}$ sites have small ${ }^{15} \mathrm{~N}$ CSA and dipolar coupling to ${ }^{1} \mathrm{H}$ is largely reduced by rotation about the three-fold 
axis. Both dips are relatively narrow. The dips at $n=1$ are broader and deeper than at $n=2$ because the first harmonic modulation component has a larger amplitude than the second harmonic. It should be noted that the amplitude distribution due to inhomogeneous $r f$ fields can affect the matching of rotary resonance conditions, particularly when the CSA is small. The experimentally observed curves are the convolution between the $r f$ field distribution and an idealized on-resonance profile. The convolution tends to reduce the depth of resonance dips, especially for the high resonance conditions because the mismatch due to $r f$ field inhomogeneity is proportional to the $r f$ field strength. The spin-lock of ${ }^{13} \mathrm{C}$ sites shows similar behavior as the ${ }^{15} \mathrm{~N}$ nuclei. The CSA rotary resonances at $v_{1 S}=n \cdot v_{\mathrm{r}}(n=1,2,3)$ are broader because of the larger ${ }^{13} \mathrm{C} \mathrm{CSA}$. The CO sites in particular (Figs. $2 \mathrm{~d}$ and $2 \mathrm{~h}$ ) have significant signal loss even for $r f$ fields far from the main $n=1$ and 2 conditions. Thus, for $\mathrm{N} \leftrightarrow \mathrm{CO}$ transfer, $r f$ fields in the range between $v_{1 S}>v_{\mathrm{r}} / 2$ and $v_{1 S}<3 \cdot v_{\mathrm{r}}$ should be avoided. 

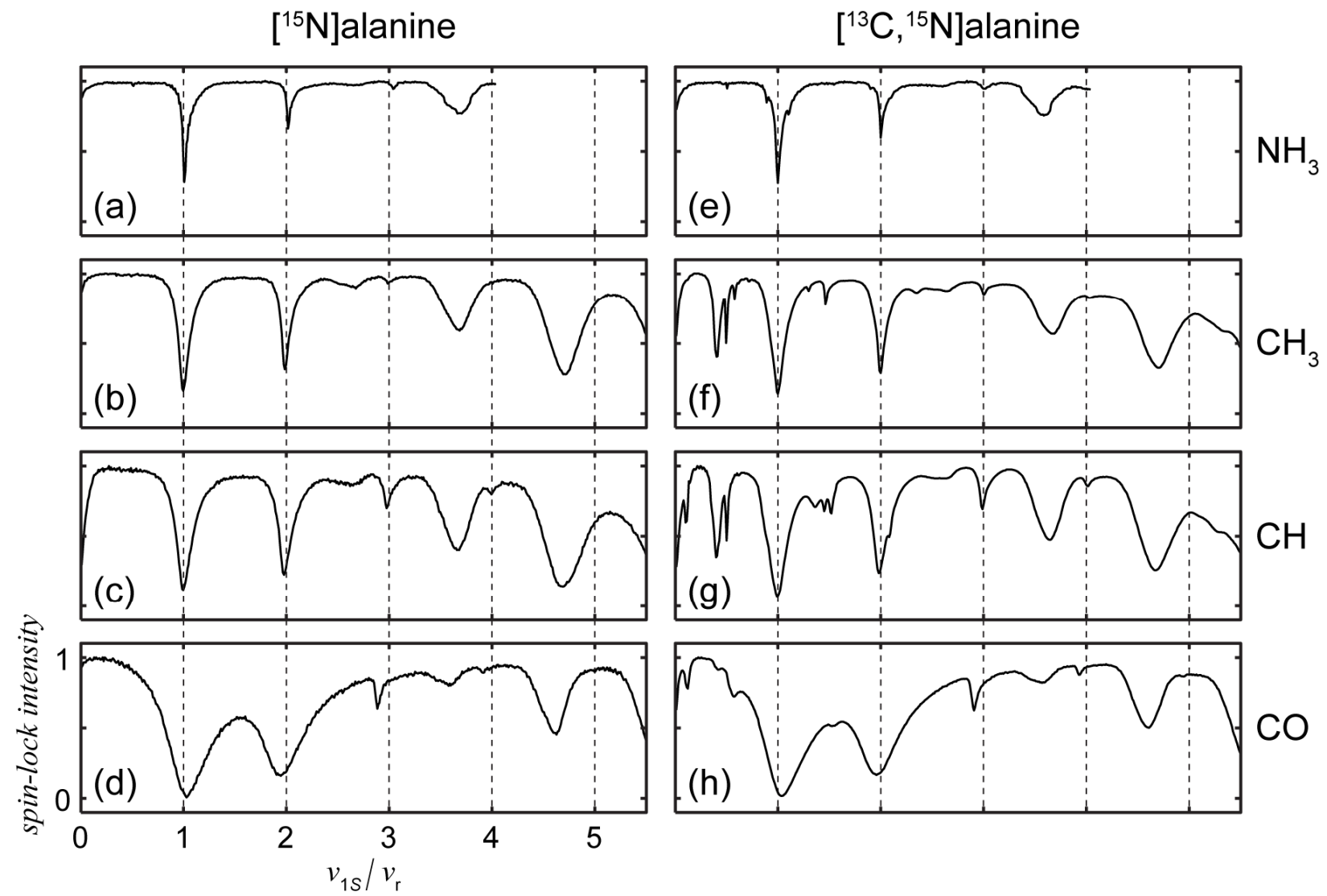

Fig. 2 Spin-lock signal intensity as a function of $r f$ field $\left(v_{1 S}\right)$ plotted in units of the MAS frequency $\left(v_{\mathrm{r}}\right)$ for (a-d) $\left[{ }^{15} \mathrm{~N}\right]$ and $(\mathrm{e}-\mathrm{h})\left[{ }^{13} \mathrm{C},{ }^{15} \mathrm{~N}\right]$ labeled alanine. Curves for the $\mathrm{NH}_{3}, \mathrm{CH}_{3}, \mathrm{CH}$ and $\mathrm{CO}$ sites are shown as labeled in the first, second, third and fourth rows, respectively. For all measurements, the transmitter frequency was put onresonance with the peak of interest labeled in the figure. ${ }^{1} \mathrm{H} \mathrm{CW}$ decoupling with $v_{1 \mathrm{H}}=100 \mathrm{kHz}$ was applied during the spin-lock, which lasted about $5 \mathrm{~ms}$ with 67 rotor periods at $v_{\mathrm{r}}=13.5 \mathrm{kHz}$.

All spin-lock curves in Fig. 2 show broad dips at the high $r f$ field end $\left(v_{1 S} / v_{\mathrm{r}}>3\right)$. These dips are separated by the spinning frequency and appear larger for sites with stronger ${ }^{1} \mathrm{H}$ heteronuclear dipolar coupling. Careful calibration of the $r f$ fields reveals high-order $\mathrm{HH}$ matching conditions between $v_{1 S}$ and $v_{1 \mathrm{H}}$, i.e., $v_{1 S}=v_{1 \mathrm{H}}-m \cdot v_{\mathrm{r}}$ at these positions. Figs. $3 \mathrm{a}-3 \mathrm{c}$ show measurements for the $\left[{ }^{15} \mathrm{~N}\right]$ alanine $\mathrm{CH}$ site using three different ${ }^{1} \mathrm{H} \mathrm{CW}$ decoupling fields $v_{1 \mathrm{H}}=$ 85,100 and $115 \mathrm{kHz}$. The results clearly show resonance dips shifting with $v_{1 \mathrm{H}}$, confirming the origin of these resonances and the associated signal loss. Resonance dips with high-order $m$ up to 5 can be seen. Efficient ${ }^{1} \mathrm{H}$ decoupling is essential for obtaining good spectral resolution and long 
$T_{2}$ relaxation times for ${ }^{13} \mathrm{C}$ and ${ }^{15} \mathrm{~N}$ nuclei. The observation of high-order ${ }^{1} \mathrm{H}-S \mathrm{CP}$ interferences highlights the importance of using strong $r f$ fields for decoupling during ${ }^{13} \mathrm{C} /{ }^{15} \mathrm{~N}$ polarization transfer. For example, at $v_{1 \mathrm{H}}=85 \mathrm{kHz}$, the $m=5{ }^{1} \mathrm{H}-{ }^{13} \mathrm{C} \mathrm{CP}$ interference near $v_{1 \mathrm{C}} \approx 1.5 v_{\mathrm{r}}$ is in the middle of the low $r f$ field region commonly used for SPECIFIC CP. Furthermore, the decoupling interference dips are fairly broad because of the relatively large $r f$ inhomogeneity proportional to the high $v_{1 \mathrm{H}}$, leaving little room to select a ${ }^{13} \mathrm{C} r f$ field for SPECIFIC CP without significant signal loss. Similar interference behavior has been observed previously [29-33], giving rise to the 'conventional wisdom' that the ${ }^{1} \mathrm{H}$ decoupling $r f$ field should be a factor of three or greater than the $r f$ fields used simultaneously on other channels.

In Figs. 3d-3f, the performance of using other ${ }^{1} \mathrm{H}$ decoupling schemes during the spinlock are examined. The SPINAL-64 [28] decoupling sequence is very effective for narrowing line widths and prolonging $T_{2}$ relaxation. Unfortunately, it worsens the performance in the case of ${ }^{13} \mathrm{C}$ spin-lock (Fig. 3d). This is not surprising because the two-pulse phase-modulation (TPPM) [34] family of decoupling schemes relies on a secondary resonance created by the small phase modulations for better averaging of ${ }^{1} \mathrm{H}$ heteronuclear dipolar coupling. A side effect of this additional mechanism is a complete loss of the ability to ${ }^{1} \mathrm{H}$ spin-lock as compared to $\mathrm{CW}$ decoupling. The phase modulation broadens the ${ }^{1} \mathrm{H} r f$ nutation frequency and consequently the ${ }^{1} \mathrm{H}-{ }^{13} \mathrm{C} \mathrm{CP}$ interferences as seen in Fig. 3d, degrading the spin-lock performance throughout the whole range. Lee-Goldburg (LG) scheme is CW decoupling with a frequency offset $\Delta v_{1 \mathrm{H}}=$ $v_{1 \mathrm{H}} / \sqrt{ } 2$ that forms an effective $r f$ field $v_{1 \mathrm{H}}{ }^{\text {eff }}$ aligned at the magic-angle $[35,36]$. Rotation about the magic-angle $\theta_{m}$ in spin space averages the ${ }^{1} \mathrm{H}$ homonuclear dipolar coupling, but heteronuclear dipolar coupling is only scaled by $\sin \left(\theta_{m}\right)$. The scaled dipolar coupling adds to the CSA modulation under MAS making the rotary resonances at $n=1$ and 2 broader (Figs. 3e and 
3f). Stronger high-order CSA rotary resonance interferences at $n=3$ and 4 are also evident with the additional C-H dipolar coupling. Nevertheless, the off-resonance decoupling increases the effective $r f$ field $v_{1 \mathrm{H}}{ }^{\text {eff }}=\sqrt{ }\left(v_{1 \mathrm{H}}{ }^{2}+\Delta v_{1 \mathrm{H}}\right)$, shifting the ${ }^{1} \mathrm{H}^{13} \mathrm{C} \mathrm{CP}$ interferences to higher $v_{1 \mathrm{C}}$. In Fig $3 \mathrm{e}, \mathrm{LG}{ }^{1} \mathrm{H}$ decoupling with $v_{1 \mathrm{H}}=82 \mathrm{kHz}$ if field $\left(v_{1 \mathrm{H}}{ }^{\text {eff }}=100 \mathrm{kHz}\right)$ was used. The result shows approximately the same ${ }^{1} \mathrm{H}-\mathrm{S} \mathrm{CP}$ interference positions as the ones with on-resonance $\mathrm{CW}{ }^{1} \mathrm{H}$ decoupling using higher $v_{1 \mathrm{H}}=100 \mathrm{kHz}$ rf field in Fig 3b. Furthermore, the LG decoupling reduces the width and depth of these interference dips. This trend can be explained by the $\sin \left(\theta_{m}\right)$ scaling to the ${ }^{1} \mathrm{H}-{ }^{13} \mathrm{C}$ flip-flop dipolar Hamiltonian responsible for the ${ }^{1} \mathrm{H}-\mathrm{S} \mathrm{CP}$ interference. The ${ }^{1} \mathrm{H}$ homonuclear decoupling by the off-resonant LG sequence also narrows the ${ }^{1} \mathrm{H}$ nutation spectrum and reduces the cross-term contributions to high-order CP. These observations suggest that LG decoupling may be advantageous in cases when sufficient ${ }^{1} \mathrm{H}$ decoupling $r f$ field is not available or when high ${ }^{13} \mathrm{C} /{ }^{15} \mathrm{~N} r f$ fields $\left(v_{1 S} / v_{\mathrm{r}}>3\right)$ are used. For low ${ }^{13} \mathrm{C} /{ }^{15} \mathrm{~N} r f$ fields, onresonance ${ }^{1} \mathrm{H} \mathrm{CW}$ decoupling still gives better spin-lock efficiency. 
$\mathrm{CW}, v_{1 \mathrm{H}}=85 \mathrm{kHz}$

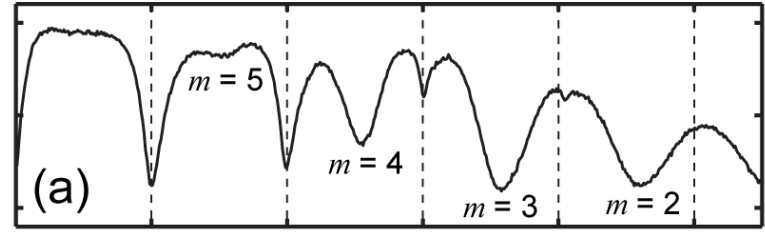

$\mathrm{CW}, v_{1 \mathrm{H}}=100 \mathrm{kHz}$
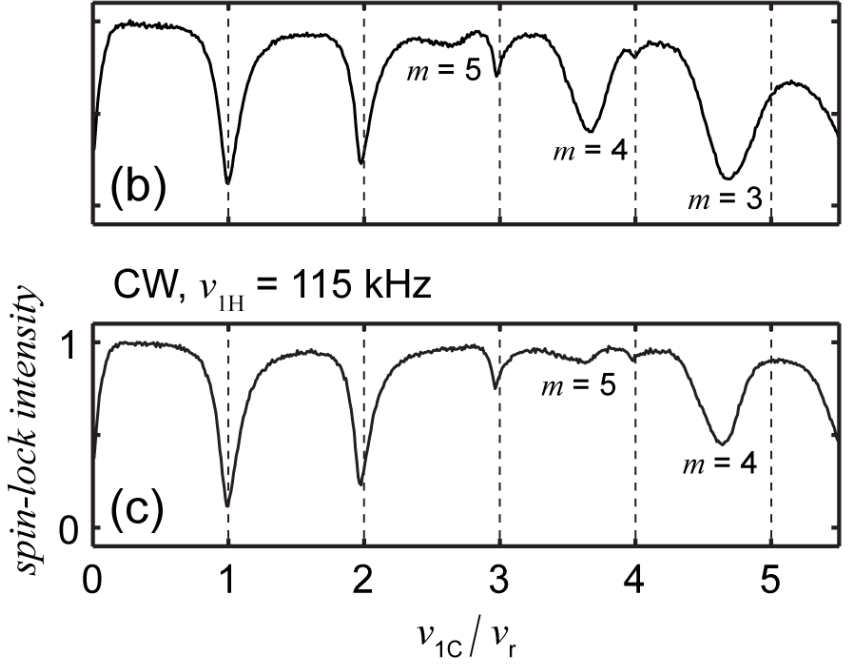

SPINAL-64, $v_{1 \mathrm{H}}=100 \mathrm{kHz}$

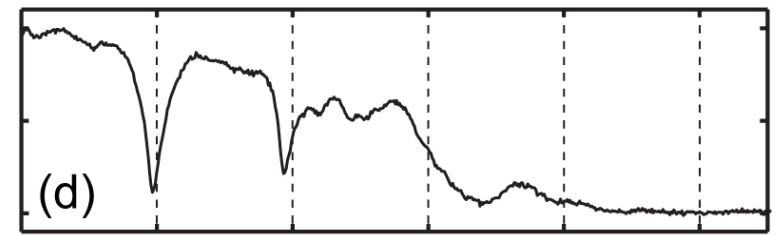

$\mathrm{LG}, v_{1 \mathrm{H}}^{\text {eff }}=100 \mathrm{kHz}$

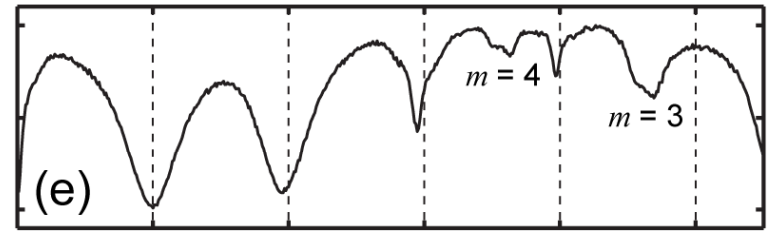

$\mathrm{LG}, v_{1 \mathrm{H}}^{\text {eff }}=122 \mathrm{kHz}$

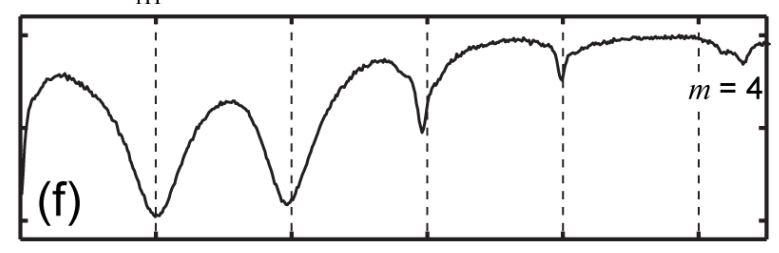

Fig. 3 Spin-lock signal intensity as a function of the ${ }^{13} \mathrm{C} r f$ field $v_{1 \mathrm{C}}$ for the $\mathrm{CH}$ site in $\left[{ }^{15} \mathrm{~N}\right]$ alanine. The methods and $r f$ fields used for ${ }^{1} \mathrm{H}$ decoupling during the spin-lock are: (a) $\mathrm{CW} v_{1 \mathrm{H}}=85 \mathrm{kHz}$, (b) $\mathrm{CW} v_{1 \mathrm{H}}=100 \mathrm{kHz}$, (c) $\mathrm{CW} v_{1 \mathrm{H}}=$ $115 \mathrm{kHz}$, (d) SPINAL-64 $v_{1 \mathrm{H}}=100 \mathrm{kHz}$, (e) LG $v_{1 \mathrm{H}}{ }^{\text {eff }}=100 \mathrm{kHz}$, and (f) LG $v_{1 \mathrm{H}}{ }^{\text {eff }}=122 \mathrm{kHz}$. For CW and LG decoupling, resonant dips caused by the ${ }^{1} \mathrm{H}$ decoupling $r f$ field are labeled with $m=\left(v_{1 \mathrm{H}}-v_{1 \mathrm{C}}\right) / v_{\mathrm{r}}$.

A side-by-side comparison of the ${ }^{13} \mathrm{C}$ spin-lock curves in Fig. 2 reveals numerous features caused by the ${ }^{13} \mathrm{C}$ homonuclear dipolar couplings. Multiple dips are visible near the halfinteger resonance conditions $n=1 / 2$ and $3 / 2$, and also at very low $r f$ fields $v_{1 \mathrm{C}} \approx 0.1 v_{\mathrm{r}}$. For a homonuclear spin pair, rotary resonance coupling occurs when the modulation frequency of the spatial part under MAS matches the nutation frequency of the bilinear spin operators of the dipolar Hamiltonian under the $r f$ field,

$$
\left|v_{1 R}^{e f f} \pm v_{1 S}^{e f f}\right|=n \cdot v_{\mathrm{r}}
$$

The effective $r f$ field $\left(v_{1 X}{ }^{e f f}\right)$ includes components from the applied $r f$ field $\left(v_{1 X}\right)$ and the frequency offset $\left(\Delta v_{X}\right)$ of the chemical to the $r f$ frequency, 


$$
v_{1 X}^{e f f}=\sqrt{v_{1 X}^{2}+\Delta v_{X}}
$$

With the large ${ }^{13} \mathrm{C}$ chemical shift range and low spin-lock $r f$ fields, adding an offset to the $r f$ field can change the resonance conditions for homonuclear dipolar recoupling. Indeed, small shifts of the resonance conditions are observed in Fig. 4 as the transmitter offset $\left(\Delta v_{\mathrm{CH}}\right)$ is changed by 1 $\mathrm{kHz}$ to either direction of the $\mathrm{CH}$ peak position.
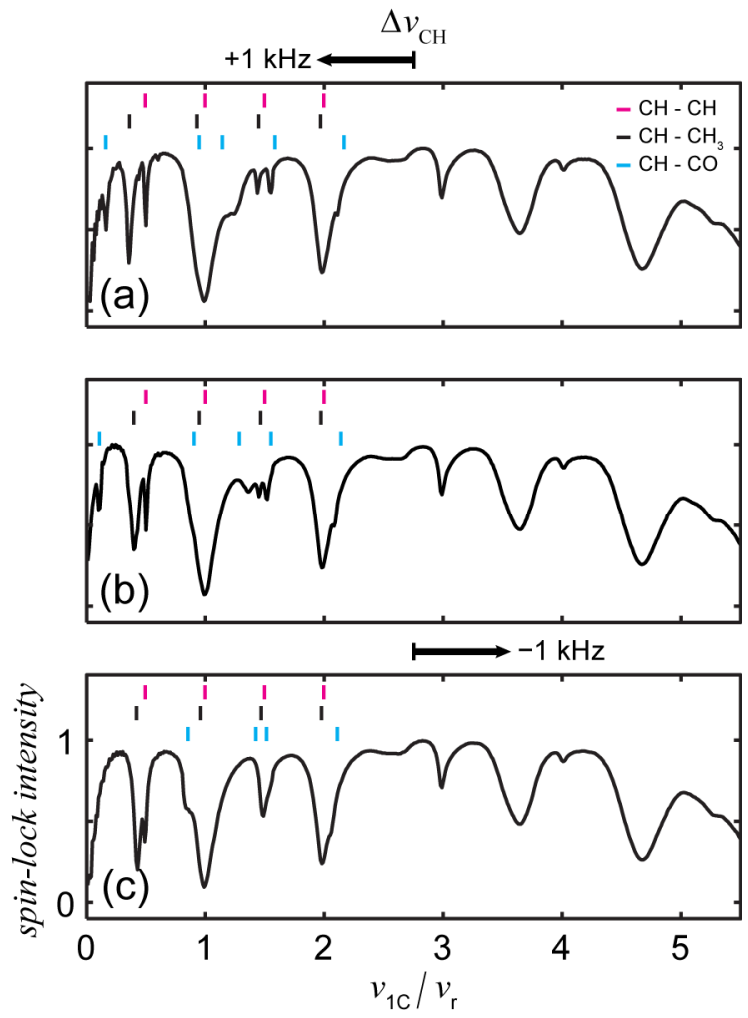

Fig. 4 Spin-lock signal intensity as a function of the ${ }^{13} \mathrm{C} r f$ field $v_{1 \mathrm{C}}$ for $\left[{ }^{13} \mathrm{C},{ }^{15} \mathrm{~N}\right]$ alanine. The transmitter frequency $\Delta v_{\mathrm{CH}}$ is positioned $+1,0$ and $-1 \mathrm{kHz}$ with respect to the $\mathrm{CH}$ peak. Calculated rotary resonance conditions due to ${ }^{13} \mathrm{C}$ homonuclear coupling between the $\mathrm{CH}$ sites and $\mathrm{CH}, \mathrm{CH}_{3}$ and $\mathrm{CO}$ sites are marked in the plots by magenta, black and cyan colored ticks, respectively.

Knowing the isotropic chemical shifts of the three ${ }^{13} \mathrm{C}$ sites in alanine (at $18.8 \mathrm{~T}, \Delta v_{\mathrm{CO}-\mathrm{CH}}$ $\left.=25516 \mathrm{~Hz}, \Delta v_{\mathrm{CH}-\mathrm{CH} 3}=6144 \mathrm{~Hz}, \Delta v_{\mathrm{CO}-\mathrm{CH} 3}=31660 \mathrm{~Hz}\right), r f$ fields that satisfy the resonance conditions in equation (1) can be calculated. The results as shown in Fig. 4 by vertical ticks are in good agreement with experimentally observed dips at the three different transmitter frequency offsets. In all cases, a dip at $v_{1 \mathrm{C}}=v_{\mathrm{r}} / 2$ is observed; this is the HORROR condition arising from 
intermolecular dipolar coupling for the on-resonance site, i.e., the $\mathrm{CH}$ site. This condition can even be seen in the ${ }^{15} \mathrm{~N}$ spin-lock curves in Fig. 2, where a miniscule dip is noticeable from the very small intermolecular ${ }^{15} \mathrm{~N}$ homonuclear dipolar coupling! The HORROR $n=1 / 2$ dip is not observed in the ${ }^{13} \mathrm{C}$ curves of $\left[{ }^{15} \mathrm{~N}\right]$ alanine because of the low $1.1 \%{ }^{13} \mathrm{C}$ natural abundance, essentially eliminating all ${ }^{13} \mathrm{C}$ homonuclear dipolar coupling.

For the three ${ }^{13} \mathrm{C}$ spins in alanine, pairwise homonuclear dipolar recoupling can occur at numerous conditions because of their chemical shift offsets. The dips slightly below $n=1 / 2$ in Fig. 4 are caused by dipolar coupling with sites that are slightly off-resonance (the $\mathrm{CH}_{3}$ site in this case) due to a small increase in the effective field $\left(v_{1 \mathrm{CH}_{3}}{ }^{\text {eff }}\right)$ from the resonance offset $\left(\Delta v_{\mathrm{CH} 3}\right)$. The dips near zero $r f$ field come from sites with large frequency offsets, namely the $\mathrm{CO}$ sites, as introducing a small $r f$ field is sufficient to satisfy the resonance condition in equation (1). Recoupling conditions near integer values of $n$ are obscured by the much larger CSA rotary resonances. The group of resonances occur near $n=3 / 2$ are clearly visible; these are high-order ${ }^{13} \mathrm{C}$ recoupling conditions that can still cause significant signal loss and should be avoided when possible. Through the whole range of $r f$ fields $v_{1 \mathrm{C}}$, it appears that only two regions around $n=0.7$ and 1.7 in the low $r f$ field regime $\left(v_{1 S} / v_{\mathrm{r}}<2\right)$ are relatively free from ${ }^{13} \mathrm{C}$ homonuclear dipolar coupling interferences for uniformly labeled samples. It should be noted that the Low- $E$ probe used for all measurements here has relatively high $r f$ field homogeneity [27]. In cases with less homogeneous $r f$ fields, the ${ }^{13} \mathrm{C}$ homonuclear coupling interferences in Fig. 4 may be broadened, but their position and consequently the optimal $r f$ fields for $\mathrm{CP}$ transfer will remain the same. 


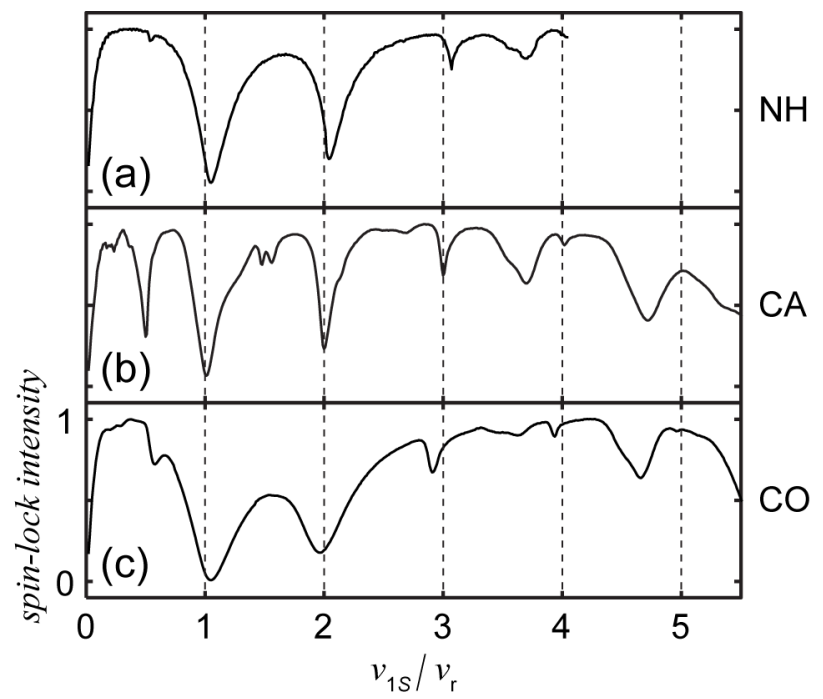

Fig. 5 Spin-lock signal intensity as a function of $r f$ field $v_{1 S}$ for the sum of all (a) NH, (b) CA, and (c) CO sites of a GB1 protein sample. The transmitter frequency was positioned at 115, 57 and $176 \mathrm{ppm}$ for the NH, CA and CO sets of peaks, respectively. ${ }^{1} \mathrm{H} \mathrm{CW}$ decoupling of $v_{1 \mathrm{H}}=100 \mathrm{kHz}$ was applied during the spin-lock, which lasted 67 rotor periods $(\sim 5 \mathrm{~ms})$ at $v_{\mathrm{r}}=13.5 \mathrm{kHz}$ MAS.

Fig. 5 shows the ${ }^{13} \mathrm{C}$ and ${ }^{15} \mathrm{~N}$ spin-lock of uniformly labeled GB1 protein. The ${ }^{15} \mathrm{~N} \mathrm{CSA}$ for amide sites is much larger than for $\mathrm{NH}_{3}$ sites; therefore the $n=1$ and 2 dips are broader (Fig. 5a) compared to the alanine samples. The dips near the $n=1 / 2$ HORROR condition are noticeable for both the $\mathrm{CO}$ and $\mathrm{NH}$ sites. The loss is particular severe for the CA signal due to the numerous ${ }^{13} \mathrm{C}$ spins of the aliphatic sidechains. The NH, CA and $\mathrm{CO}$ curves in Fig. 5 provide guidelines for selecting $r f$ fields applicable to protein backbone ${ }^{13} \mathrm{C} \leftrightarrow{ }^{15} \mathrm{~N} \mathrm{CP}$ transfers. Both $r f$ fields should be in regions with good spin-lock and their difference (or sum) should match the spinning frequency (i.e., $\left|v_{1 \mathrm{C}} \pm v_{1 \mathrm{~N}}\right|=v_{\mathrm{r}}$ ) for ZQ (or DQ) CP. The first sideband of the $\mathrm{HH}$ condition is chosen for its larger amplitude. With these considerations in mind, the following conditions have been found suitable for $\mathrm{N} \leftrightarrow \mathrm{CO}$ and $\mathrm{N} \leftrightarrow \mathrm{CA}$ transfer. It should be noted that the optimal conditions are strongly dependent on the spinning frequency and the available ${ }^{1} \mathrm{H}$ decoupling $r f$ fields. The specific conditions presented here are for a $13.5 \mathrm{kHz}$ MAS frequency, 
$1 \mathrm{H} \mathrm{CW}$ decoupling in the range of approximately $100 \mathrm{kHz}$ and an external magnetic field of 18.8 $\mathrm{T}, v_{0}\left({ }^{1} \mathrm{H}\right)=800 \mathrm{MHz}$.

For $\mathrm{N} \leftrightarrow \mathrm{CO}$ coherence transfer, both the $\mathrm{CO}$ and $\mathrm{NH}$ sites have large CSA, which give rise to broad CSA rotary resonance dips at $v_{1 S}=n \cdot v_{\mathrm{r}}(n=1,2)$. Therefore, the typical conditions used for ZQ NCO transfer are $v_{1 \mathrm{C}}=3.5 v_{\mathrm{r}}$ and $v_{1 \mathrm{~N}}=2.5 v_{\mathrm{r}}$, matching the first sideband of the HH condition. There are two reasons to apply the higher $r f$ field on the $\mathrm{CO}$ sites. First, the $\mathrm{CO}$ sites have larger CSA in $\mathrm{Hz}$ than the NH sites, so the CO CSA interferences are broader and can cause more signal loss at $v_{1 S} / v_{\mathrm{r}}=2.5$. Second, the ${ }^{1} \mathrm{H}$ heteronuclear dipolar coupling is stronger for $\mathrm{NH}$ than CO sites; thus, using a lower ${ }^{15} \mathrm{~N}$ rf field can help avoid interference with the ${ }^{1} \mathrm{H}$ decoupling. Even for the CO sites, there is noticeable dips of signal loss near the $v_{1 \mathrm{C}}=3.5 v_{\mathrm{r}}$ condition from the $m=4$ high-order $\mathrm{CP}$ interference with the $100 \mathrm{kHz}{ }^{1} \mathrm{H}$ decoupling $r f$ field in Fig. 5a. A possibly better condition for $\mathrm{N} \leftrightarrow \mathrm{CO}$ transfer can be found for DQ CP at low $r f$ fields, $v_{1 S} / v_{\mathrm{r}}<1$, where the spin-lock for both the $\mathrm{CO}$ and $\mathrm{NH}$ sites performs well (Figs. 5a and 5c). Using $v_{1 \mathrm{C}}=$ $0.35 v_{\mathrm{r}}$ for CO sites and $v_{1 \mathrm{~N}}=0.65 v_{\mathrm{r}}$ for NH sites, the condition for ${ }^{15} \mathrm{~N} \leftrightarrow{ }^{13} \mathrm{C}$ DQ CP transfer can be satisfied.

For $\mathrm{N} \leftrightarrow \mathrm{CA}$ coherence transfer, the interference due to ${ }^{13} \mathrm{C}$ CSA rotary resonance is much smaller (Fig. 5b). A narrow range near $v_{1 \mathrm{C}}=1.7 v_{\mathrm{r}}$ gives good spin-lock. The ${ }^{15} \mathrm{~N} \leftrightarrow{ }^{13} \mathrm{C}$ ZQ CP condition requires that the ${ }^{15} \mathrm{~N} r f$ field be $v_{1 \mathrm{~N}}=2.7 v_{\mathrm{r}}$. This $r f$ setting is sufficiently away from the $n=2$ CSA resonance and hence should provide good spin-lock efficiency. It is also possible to increase both $r f$ fields by $v_{\mathrm{r}}$ (i.e., $v_{1 \mathrm{C}}=2.7 v_{\mathrm{r}}$ and $v_{1 \mathrm{~N}}=3.7 v_{\mathrm{r}}$ ), though the higher $r f$ fields may start to enter the ${ }^{1} \mathrm{H}$ decoupling interference regime. $\mathrm{A}{ }^{15} \mathrm{~N} r f$ field of $v_{1 \mathrm{~N}}=3.7 v_{\mathrm{r}}$ may also become demanding and hard to achieve for the low- $\gamma^{15} \mathrm{~N}$ nucleus. At low $r f$ fields, good 
spin-lock can be found for the combination $v_{1 \mathrm{C}}=0.65 v_{\mathrm{r}}$ and $v_{1 \mathrm{~N}}=0.35 v_{\mathrm{r}}$, satisfying the first sideband for ${ }^{15} \mathrm{~N} \rightarrow{ }^{13} \mathrm{C}$ DQ CP transfer.

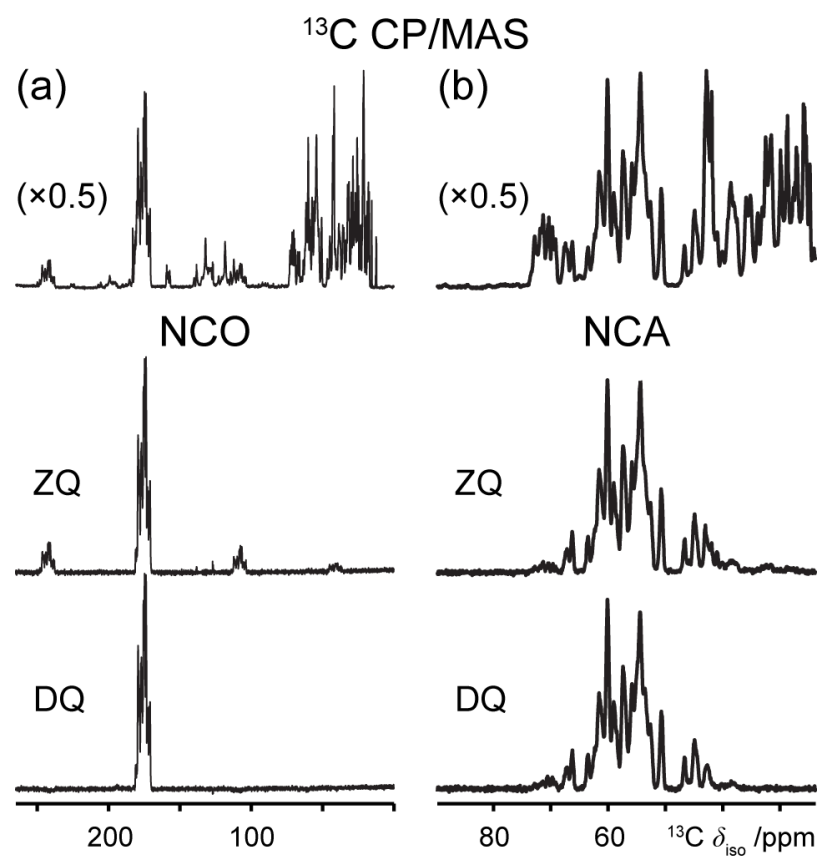

Fig. 6 Comparison of ${ }^{1} \mathrm{H} \rightarrow{ }^{13} \mathrm{C}$ and ${ }^{1} \mathrm{H} \rightarrow{ }^{15} \mathrm{~N} \rightarrow{ }^{13} \mathrm{C} \mathrm{CP}$ spectra of GB1. The $r f$ fields used were: ZQ NCO $\left\{v_{1 \mathrm{C}}=\right.$ $\left.3.5 v_{\mathrm{r}}, v_{1 \mathrm{~N}}=2.5 v_{\mathrm{r}}\right\}$, ZQ NCA $\left\{v_{1 \mathrm{C}}=1.7 v_{\mathrm{r}}, v_{1 \mathrm{~N}}=2.7 v_{\mathrm{r}}\right\}$, DQ NCO $\left\{v_{1 \mathrm{C}}=0.35 v_{\mathrm{r}}, v_{1 \mathrm{~N}}=0.65 v_{\mathrm{r}}\right\}$, and DQ NCA $\left\{v_{1 \mathrm{C}}=\right.$ $\left.0.65 v_{\mathrm{r}}, v_{1 \mathrm{~N}}=0.35 v_{\mathrm{r}}\right\}$. For both ZQ and DQ CP transfer, $\pm \sim 1$ and $\pm \sim 1.5 \mathrm{kHz}$ linear ramps were applied to the ${ }^{15} \mathrm{~N}$ channel for the NCA and NCO spectra, respectively. All other experimental parameters are the same as in Fig. 5.

Comparisons of the ${ }^{1} \mathrm{H} \rightarrow{ }^{15} \mathrm{~N} \rightarrow{ }^{13} \mathrm{C}$ double $\mathrm{CP}$ spectra with direct ${ }^{1} \mathrm{H} \rightarrow{ }^{13} \mathrm{C} \mathrm{CP}$ spectra are shown in Fig. 6 for the $\mathrm{CO}$ and $\mathrm{CA}$ sites of GB1 protein. The intensity ratio between the two spectra obtained under identical experimental conditions is an indicator of the ${ }^{15} \mathrm{~N} \rightarrow{ }^{13} \mathrm{C}$ transfer efficiency, assuming that the first CP step from the protons reaches the same level of polarization. Two sets of 1D NCO and NCA spectra are shown in Fig. 6, one acquired with ZQ and the other with DQ ${ }^{15} \mathrm{~N} \rightarrow{ }^{13} \mathrm{C} \mathrm{CP}$ using the optimal ${ }^{15} \mathrm{~N}$ and ${ }^{13} \mathrm{C} r f$ fields discussed above. The ZQ and DQ spectra display nearly identical efficiencies of $\sim 55 \%$ for NCO transfer and $\sim 45 \%$ for NCA transfer. Their main differences are the absence of spinning sidebands in the NCO spectrum and smaller aliphatic sidechain signals in the $<40 \mathrm{ppm}$ region in the NCA 
spectrum when employing DQ CP. The low $r f$ fields make the transfers more selective in terms of the frequency offset but still sufficient for covering the ${ }^{13} \mathrm{C}$ isotropic chemical shift range of both $\mathrm{CO}$ and $\mathrm{CA}$ sites without noticeable intensity distortion. As further evidence of the viability of using ${ }^{15} \mathrm{~N} \leftrightarrow{ }^{13} \mathrm{C}$ DQ CP transfer, 2D NCO and NCA spectra of the GB1 protein using the two different CP conditions (ZQ and DQ) are shown in Fig. 7 along with their corresponding ${ }^{13} \mathrm{C}$ and ${ }^{15} \mathrm{~N}$ projections; the ZQ and DQ spectra are nearly identical.
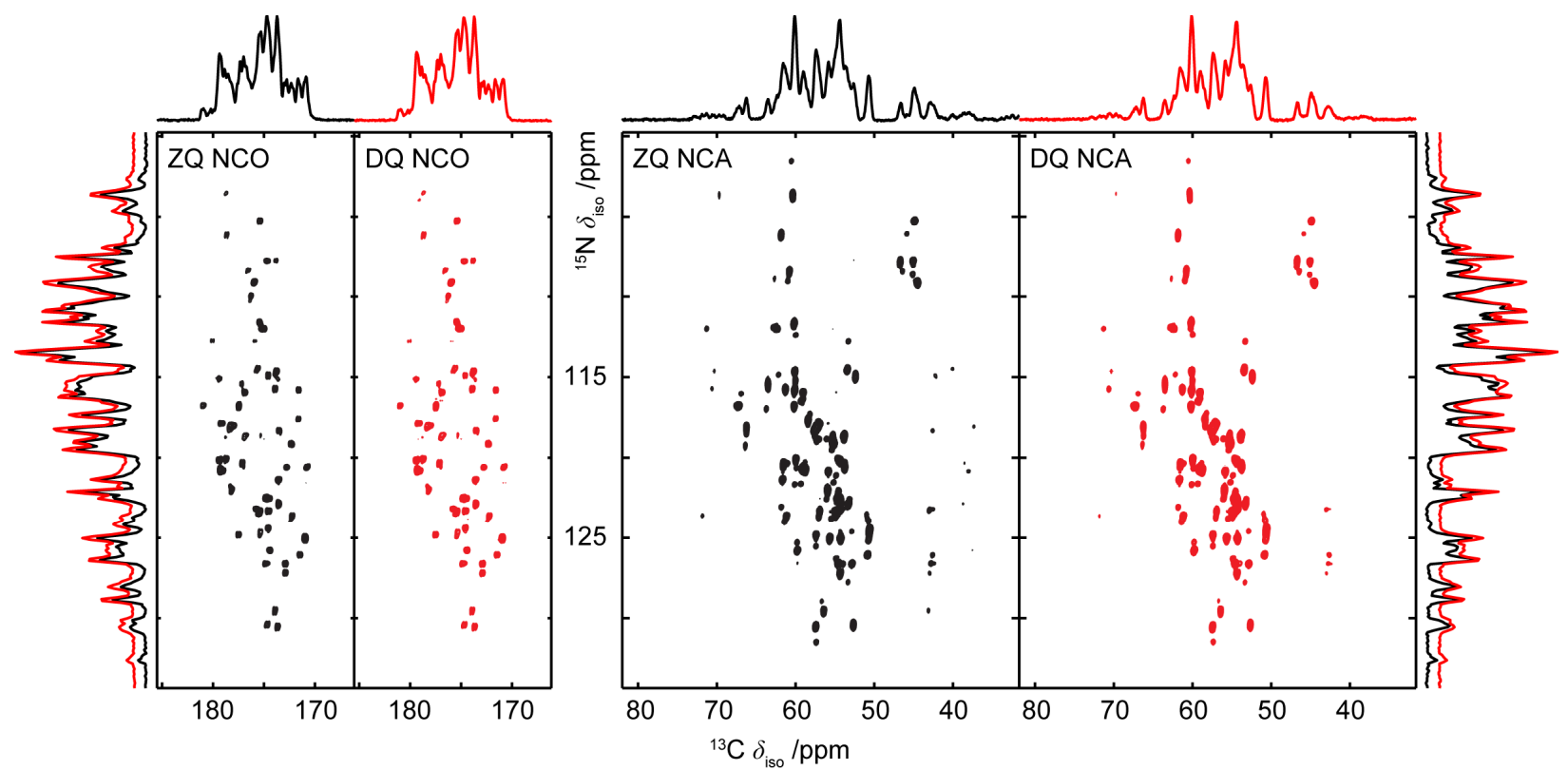

Fig. 7 Comparison of 2D NCO and NCA spectra along with sum projections of GB1 protein acquired using ZQ (black traces) and DQ (red traces) ${ }^{15} \mathrm{~N} \rightarrow{ }^{13} \mathrm{C} \mathrm{CP}$. The maximum acquisition time was $40 \mathrm{~ms}$ for both $t_{1}$ and $t_{2}$ evolutions. Other experimental conditions are the same as in Fig. 6.

The NCO and NCA spectra in Fig. 6 were obtained by applying a linear amplitude ramp to the ${ }^{15} \mathrm{~N}$ spin-lock pulse, and the signal intensities were optimized by varying the mean $r f$ power and the amplitude range of the ramp experimentally. The amplitude ramp was applied to the ${ }^{15} \mathrm{~N}$ channel since there are fewer interference phenomena observed for the NH sites (Fig. 5a) and thus less propensity for stepping into interference regions when varying $v_{1 \mathrm{~N}}$. Other more sophisticated ramps such as tangential amplitude modulation can also be used but generally require optimization of more parameters. The amplitude ramp enhances the transfer efficiency 
through two mechanisms. First, it compensates mismatch to the HH conditions due to $r f$ inhomogeneity. Second, a slow adiabatic passage across the HH condition can ideally invert the polarization between the two spins instead of bringing them to equilibrium, where the polarization is distributed in half. The observed NCO transfer efficiency of $\sim 55 \%$ obtained for GB1, under an undetermined amount of loss due to $T_{1 \rho}$ relaxation, indicates some contribution from the adiabatic mechanism. The optimized spectra in Fig. 6 were obtained with the following ${ }^{15} \mathrm{~N}$ ramp amplitude limits: $v_{1 \mathrm{~N}} \pm \sim 1 \mathrm{kHz}$ for NCA, and $v_{1 \mathrm{~N}} \pm \sim 1.5 \mathrm{kHz}$ for NCO, for both ZQ and DQ CP. These values are qualitatively in the range of the ${ }^{13} \mathrm{C}-{ }^{15} \mathrm{~N}$ dipolar coupling plus the estimated amplitude of the $\mathrm{HH}$ mismatch due to $r f$ field inhomogeneity. For the $\mathrm{CO}$ site, a larger amplitude ramp is required to compensate the contribution of the CSA modulation to the effective ${ }^{13} \mathrm{C}$ nutation frequency.

\section{Conclusion}

It has been shown in this work that for uniformly ${ }^{13} \mathrm{C}$ and ${ }^{15} \mathrm{~N}$ labeled samples the chemical shift anisotropy, and ${ }^{1} \mathrm{H}$ heteronuclear and ${ }^{13} \mathrm{C}$ homonuclear dipolar couplings can deteriorate the spin-lock of ${ }^{13} \mathrm{C}$ and ${ }^{15} \mathrm{~N}$ nuclei under MAS. Loss of polarization occurs when the spin-lock $r f$ field encounters various resonant interference conditions relative to the spinning frequency, $n=v_{1} / v_{\mathrm{r}}$. The location and physical origin of these conditions have been identified. In particular, the spin-lock $r f$ fields should always avoid the CSA rotary resonance conditions at integer $n=1,2$ and higher. Interference with ${ }^{1} \mathrm{H}$ decoupling can occur at high-order sidebands of the HH condition $m=\left(v_{1 \mathrm{H}}-v_{1 \mathrm{C}}\right) / v_{\mathrm{r}}$ with $m$ up to 5 and can cause signal loss when high spin-lock $r f$ fields are used. This can be partially remedied by employing off-resonance LG decoupling when sufficiently high ${ }^{1} \mathrm{H} r f$ fields are not available. Interference from ${ }^{13} \mathrm{C}$ homonuclear dipolar 
coupling occurs near the $n=1 / 2$ and 3/2 conditions and also at very low $r f$ fields near $n \sim 0.1$. Sets of ${ }^{13} \mathrm{C} /{ }^{15} \mathrm{~N} r f$ fields that avoid loss of spin-lock efficiency from all these interactions are found for both the zero- and double-quantum ${ }^{13} \mathrm{C} \leftrightarrow{ }^{15} \mathrm{~N}$ coherence transfer. Demonstrated using a model protein sample, double-quantum $\mathrm{CP}$ using $r f$ fields lower than $v_{\mathrm{r}}$ can achieve more than $50 \%$ efficiency and is as efficient as more commonly used zero-quantum CP conditions. The conditions for all the interferences and optimal selection of $r f$ fields for polarization transfer shown here depend strongly on the chosen spinning frequency and available ${ }^{1} \mathrm{H}$ decoupling $r f$ field. For experimental conditions different from those used in this study (13.5 kHz MAS, 100 $\mathrm{kHz}{ }^{1} \mathrm{H} \mathrm{CW}$ decoupling $r f$ field, $\left.v_{0}\left({ }^{1} \mathrm{H}\right)=800 \mathrm{MHz}\right)$ a careful mapping of the interferences should be performed to select $r f$ fields optimal for the important ${ }^{13} \mathrm{C} \leftrightarrow{ }^{15} \mathrm{~N}$ coherence transfer step(s) in many multi-dimensional MAS experiments of proteins.

\section{Acknowledgements}

This work was performed at the National High Magnetic Field Laboratory, which is supported by NSF DMR-1157490 and the State of Florida. 


\section{References}

[1] A. Abragam, The Principles of Nuclear Magnetism, Academic Press, New York, 1962.

[2] S.R. Hartmann, E.L. Hahn, Nuclear Double Resonance in Rotating Frame, Physical Review 128 (1962) 2042-2053.

[3] A. Pines, M.G. Gibby, J.S. Waugh, Proton-Enhanced Nmr of Dilute Spins in Solids, J. Chem. Phys. 59 (1973) 569-590.

[4] J. Schaefer, E.O. Stejskal, C-13 Nuclear Magnetic-Resonance of Polymers Spinning at Magic Angle, J. Am. Chem. Soc. 98 (1976) 1031-1032.

[5] M. Baldus, A.T. Petkova, J. Herzfeld, R.G. Griffin, Cross polarization in the tilted frame: assignment and spectral simplification in heteronuclear spin systems, Mol. Phys. 95 (1998) 1197-1207.

[6] A.T. Petkova, M. Baldus, M. Belenky, M. Hong, R.G. Griffin, J. Herzfeld, Backbone and side chain assignment strategies for multiply labeled membrane peptides and proteins in the solid state, J. Magn. Reson. 160 (2003) 1-12.

[7] E.O. Stejskal, J. Schaefer, J.S. Waugh, Magic-Angle Spinning and Polarization Transfer in Proton-Enhanced Nmr, J. Magn. Reson. 28 (1977) 105-112.

[8] M. Sardashti, G.E. Maciel, Effects of Sample Spinning on Cross Polarization, J. Magn. Reson. 72 (1987) 467-474.

[9] R.A. Wind, S.F. Dec, H. Lock, G.E. Maciel, C-13 Cp Mas and High-Speed Magic-Angle Spinning, J. Magn. Reson. 79 (1988) 136-139.

[10] B.H. Meier, Cross Polarization under Fast Magic Angle Spinning - Thermodynamical Considerations, Chem. Phys. Lett. 188 (1992) 201-207.

[11] S. Laage, A. Marchetti, J. Sein, R. Pierattelli, H.J. Sass, S. Grzesiek, A. Lesage, G. Pintacuda, L. Emsley, Band-Selective H-1-C-13 Cross-Polarization in Fast Magic Angle Spinning Solid-State NMR Spectroscopy, J. Am. Chem. Soc. 130 (2008) 17216-17217.

[12] A. Lange, I. Scholz, T. Manolikas, M. Ernst, B.H. Meier, Low-power cross polarization in fast magic-angle spinning NMR experiments, Chem. Phys. Lett. 468 (2009) 100-105.

[13] J.P. Demers, V. Vijayan, S. Becker, A. Lange, Tailored low-power cross-polarization under fast magic-angle spinning, J. Magn. Reson. 205 (2010) 216-223.

[14] G. Metz, X.L. Wu, S.O. Smith, Ramped-Amplitude Cross-Polarization in Magic-AngleSpinning Nmr, J. Magn. Reson. Ser. A 110 (1994) 219-227.

[15] S. Hediger, B.H. Meier, N.D. Kurur, G. Bodenhausen, R.R. Ernst, Nmr Cross-Polarization by Adiabatic Passage through the Hartmann-Hahn Condition (Aphh), Chem. Phys. Lett. 223 (1994) 283-288.

[16] S. Hediger, B.H. Meier, R.R. Ernst, Adiabatic Passage Hartmann-Hahn Cross-Polarization in Nmr under Magic-Angle Sample-Spinning, Chem. Phys. Lett. 240 (1995) 449-456.

[17] R.Q. Fu, S.A. Smith, G. Bodenhausen, Recoupling of heteronuclear dipolar interactions in solid state magic-angle spinning NMR by simultaneous frequency and amplitude modulation, Chem. Phys. Lett. 272 (1997) 361-369.

[18] K.J. Harris, A. Lupulescu, B.E.G. Lucier, L. Frydman, R.W. Schurko, Broadband adiabatic inversion pulses for cross polarization in wideline solid-state NMR spectroscopy, J. Magn. Reson. 224 (2012) 38-47.

[19] S. Wi, Z. Gan, R.W. Schurko, L. Frydman, Cross-polarization phenomena in the NMR of fast spinning solids subject to adiabatic sweeps, J. Chem. Phys. 142 (2015) 064201. 
[20] A. Brinkmann, M.H. Levitt, Symmetry principles in the nuclear magnetic resonance of spinning solids: Heteronuclear recoupling by generalized Hartmann-Hahn sequences, J. Chem. Phys. 115 (2001) 357-384.

[21] C. Kehlet, M. Bjerring, A.C. Sivertsen, T. Kristensen, J.J. Enghild, S.J. Glaser, N. Khaneja, N.C. Nielsen, Optimal control based NCO and NCA experiments for spectral assignment in biological solid-state NMR spectroscopy, J. Magn. Reson. 188 (2007) 216-230.

[22] D.P. Raleigh, M.H. Levitt, R.G. Griffin, Rotational Resonance in Solid-State Nmr, Chem. Phys. Lett. 146 (1988) 71-76.

[23] T.G. Oas, R.G. Griffin, M.H. Levitt, Rotary Resonance Recoupling of Dipolar Interactions in Solid-State Nuclear Magnetic-Resonance Spectroscopy, J. Chem. Phys. 89 (1988) 692695.

[24] Z.H. Gan, D.M. Grant, Rotational Resonance in a Spin-Lock Field for Solid-State Nmr, Chem. Phys. Lett. 168 (1990) 304-308.

[25] N.C. Nielsen, H. Bildsoe, H.J. Jakobsen, M.H. Levitt, Double-Quantum Homonuclear Rotary Resonance - Efficient Dipolar Recovery in Magic-Angle-Spinning NuclearMagnetic-Resonance, J. Chem. Phys. 101 (1994) 1805-1812.

[26] R. Verel, M. Baldus, M. Ernst, B.H. Meier, A homonuclear spin-pair filter for solid-state NMR based on adiabatic-passage techniques, Chem. Phys. Lett. 287 (1998) 421-428.

[27] S.A. McNeill, P.L. Gor'kov, K. Shetty, W.W. Brey, J.R. Long, A low-E magic angle spinning probe for biological solid state NMR at 750 MHz, J. Magn. Reson. 197 (2009) 135-144.

[28] B.M. Fung, A.K. Khitrin, K. Ermolaev, An improved broadband decoupling sequence for liquid crystals and solids, J. Magn. Reson. 142 (2000) 97-101.

[29] Y. Ishii, J. Ashida, T. Terao, C-13-H-1 Dipolar Recoupling Dynamics in C-13 MultiplePulse Solid-State Nmr, Chem. Phys. Lett. 246 (1995) 439-445.

[30] I. Marin-Montesinos, D.H. Brouwer, G. Antonioli, W.C. Lai, A. Brinkmann, M.H. Levitt, Heteronuclear decoupling interference during symmetry-based homonuclear recoupling in solid-state NMR, J. Magn. Reson. 177 (2005) 307-317.

[31] M.J. Bayro, R. Ramachandran, M.A. Caporini, M.T. Eddy, R.G. Griffin, Radio frequencydriven recoupling at high magic-angle spinning frequencies: Homonuclear recoupling sans heteronuclear decoupling, J. Chem. Phys. 128 (2008) 052321.

[32] J.R. Lewandowski, G. De Paepe, M.T. Eddy, J. Struppe, W. Maas, R.G. Griffin, Proton Assisted Recoupling at High Spinning Frequencies, J. Phys. Chem. B 113 (2009) 90629069.

[33] G. De Paepe, J.R. Lewandowski, A. Loquet, M. Eddy, S. Megy, A. Bockmann, R.G. Griffin, Heteronuclear proton assisted recoupling, J. Chem. Phys. 134 (2011) 095101.

[34] A.E. Bennett, C.M. Rienstra, M. Auger, K.V. Lakshmi, R.G. Griffin, Heteronuclear Decoupling in Rotating Solids, J. Chem. Phys. 103 (1995) 6951-6958.

[35] W.I. Goldburg, M. Lee, Nuclear Magnetic Resonance Line Narrowing by a Rotating Rf Field, Phys. Rev. Lett. 11 (1963) 255-258.

[36] M. Lee, W.I. Goldburg, Nuclear-Magnetic-Resonance Line Narrowing by a Rotating Rf Field, Physical Review 140 (1965) 1261-1271. 
- Interferences from CSA, heteronuclear and homonuclear dipolar coupling to spin-lock under magic-angle spinning are presented.

- Optimal $r f$ fields for ${ }^{13} \mathrm{C} \leftrightarrow{ }^{15} \mathrm{~N}$ coherence transfer are selected based on spin-lock efficiency. $-{ }^{13} \mathrm{C} \leftrightarrow{ }^{15} \mathrm{~N}$ double-quantum $\mathrm{CP}$ provides equal efficiency, but better selectivity compared with zero-quantum $\mathrm{CP}$ transfer. 